\title{
A Metal-Insulator Transition for the Almost Mathieu Model
}

\author{
J. Bellissard $\star$ R. Lima, and D. Testard ${ }^{\star \star}$ \\ Centre de Physique Théorique, CNRS, F-13288 Marseille Cedex 9, France
}

\begin{abstract}
We study the spectrum of the almost Mathieu hamiltonian:

$$
\left(H_{x} \psi\right)(n)=\psi(n+1)+\psi(n-1)+2 \mu \cos (x-n \theta) \psi(n), \quad n \in \mathbb{Z},
$$

where $\theta$ is an irrational number and $x$ is in the circle $\mathbb{T}$. For a small enough coupling constant $\mu$ and any $x$ there is a closed energy set of non-zero measure in the absolutely continuous spectrum of $H$. For sufficiently high $\mu$ and almost all $x$ we prove the existence of a set of eigenvalues whose closure has positive measure. The two results are obtained for a subset of irrational numbers $\theta$ of full Lebesgue measure.
\end{abstract}

\section{Introduction}

The aim of this paper is to study some properties of the spectrum of operators of the form:

$$
H_{x}^{(\mu)} \psi(n)=\psi(n+1)+\psi(n-1)+\mu V(x-n \theta) \psi(n),
$$

where $\psi \in \ell^{2}(\mathbb{Z}), V$ is a continuous function on the circle $\mathbb{T}, \theta$ is an irrational number, $x \in \mathbb{T}$ and $\mu$ is a real positive number (the coupling constant). From the physical point of view, both the dependence of the spectrum on $\mu$, as well as the growth of the eigenfunctions as $n \rightarrow \infty$ are crucial.

The first example of the treatment of an almost periodic potential goes back to Peierls [21] where the Schrödinger operator defined in (1.1) describes the one band hamiltonian for a Bloch electron in a magnetic field, in the approximation where the interband contributions is neglected; see also [22]. The prediction of Little

* Also at: Université de Provence

$\star \star$ Also at: Centre Universitaire d'Avignon 
[19] concerning the possibility of getting supraconductivity at high temperatures using organic conductors gave, more recently, a new interest to the subject.

Subsequently a great effort was made theoretically as well as experimentally in these fields. The first studied materials, such as the family TTF-TCNQ [8], failed to be conductors below $40 \mathrm{~K}$ since they present a metal-insulator transition, due to the Peierls instability. In order to avoid Peierls instability, one has to increase the rigidity of the material. This was performed very recently using the (TMTSF)-PF 6 ; [7], for which material it seems that supraconductivity can appear at higher temperatures.

On the other hand, Fröhlich theory [11] predicts superconductivity provided the perturbing modulation is small enough. It has been conjectured that large modulation creates localization of the electrons as argued by Anderson [1] for the case of random media.

One of the main heuristic steps in the understanding of the phenomenum is due to Aubry and André [2], who proposed a tight binding approximation, for which the Schrödinger operator, known as the almost Mathieu operator, consists of the operator (1.1) in the case where $V(y)=2 \cos 2 \pi y$. Computing the Liapounov exponent $\gamma$ which gives the asymptotic behaviour of $\psi(n)$ as $n \rightarrow \infty$, they found that for almost all $x$

$$
\gamma \geqq \log |\mu|
$$

suggesting localization properties for $\mu>1$. An argument, known as the AndréAubry duality [2] relates the spectra of $H_{x}^{(\mu)}$ and $H_{x}^{(1 / \mu)}$ suggesting then a conducting phase for $\mu<1$. See Avron and Simon [4] for a rigorous proof of the Thouless formula and (1.2). Subsequently Hermann [16] gave a simpler proof of (1.2). However, as it was recently pointed out by Avron and Simon [4] a result of Gordon [12] implies that localization cannot occur for all irrational values of $\theta$.

Therefore at most we can expect that for "almost" all irrational $\theta$, the metalinsulator transition really takes place. The aim of this paper is to prove rigorously that such is the case.

There are two pieces of mathematical machinery which will be employed. First we use the method developed by Dinaburg and Sinai [9] for studying the spectrum of $H_{x}^{(\mu)}$ for small values of $\mu$. This method is based on the Newton algorithm for computing roots of certain equations, together with the analysis of the small divisors as in the classical work of Kolmogoroff [18], Arnold [3], and Moser [20]. Actually we use Rüssmann's point of view [23] with the main difference coming in our case from the fact that we treat a discretized version of the Schrödinger equation.

After that, we use, in an essential way the André-Aubry duality. In a previous paper [6], two of us showed that this duality is fairly general, since it appears as a Fourier transform in the algebraic formalism related to this problem. In our work we use this duality argument in order to show the existence of an infinite number of eigenvalues, with corresponding exponentially localized eigenfunctions. Furthermore we are able to prove, using techniques of number theory, that the measure of the closure of the set of eigenvalues is strictly positive. 
The paper is organized as follows. In Sect. 2, we recall some useful results about number theory and Rüssmann approximation functions, and we fix some functional spaces used in the sequel. Section 3 contains the list of results. In Sect. 4 we make the first steps of an application of the Dinaburg-Sinai method using Rüssmann's point of view and obtain estimates of the linearized equation. In Sect. 5 we achieve the proof of Theorem 1 inspecting a recursion process. The existence of absolutely continuous spectrum for small coupling, which is the content of Theorem 2, is proved in Sect. 6. Finally, the proof of the existence of a pure point spectrum at high coupling, together with an estimation of its Lebesgue measure, is given in Sect. 7. Section 8 is devoted to the case of a special type of irrationals $\theta$ for which the estimate of the size of the spectrum can be improved.

Remark. Some of the results contained in this paper were announced by one of us (J.B.) at the Summer Institute on Operator Algebras and Applications, Kingston (Ontario), July 1980, and the VIth International Conference on Mathematical Physics, Berlin, August 1981.

\section{Preliminaries}

Here we fix some notation and recall some results used in the sequel.

\subsection{Rüssmann's Approximation Functions [23]}

An RAF (Rüssmann approximation function) is a strictly positive function $\Omega$ on $[0,+\infty[$ which satisfies:

i) $\Omega$ is continuous, decreasing and

ii) $\begin{gathered}s \rightarrow \frac{1}{s} \log \frac{1}{\Omega(s)} \text { is decreasing, } \\ \text { iii) } \quad \int_{s_{0}}^{+\infty} d s \frac{1}{s^{2}} \log \frac{1}{\Omega(s)}<+\infty \text { for any } s_{0}>0 .\end{gathered}$

A useful example of an RAF is given by

$$
\begin{aligned}
& \Omega(s)=\Omega\left(s_{0}\right) \quad \text { if } \quad s \leqq s_{0}=e^{1+\alpha}, \\
& \Omega(s)=C \exp -s /(\log s)^{1+\alpha} \text { for } s \geqq s_{0},
\end{aligned}
$$

where $\alpha \leqq \frac{1}{2}$ and $C$ are strictly positive constants.

Clearly the product of two RAF's is again an RAF, so that in particular positive powers of an RAF are RAF's. Following [23], we now introduce the functions

$$
\Phi_{m}(\varrho)=\int_{0}^{\infty} \frac{e^{-s}}{\Omega(s / \varrho)^{m}} d s \quad \text { for } \varrho>0
$$


Here $\Phi_{m}$ is a decreasing function of $\varrho$. Further we define $D S(\varrho)$ to be the set of positive decreasing sequences $\underline{r}=\left(r_{n}\right)_{n \geqq 0}$ such that $\sum_{n=0}^{\infty} r_{n} \leqq \varrho$. Endowed with the topology of pointwise convergence, $\operatorname{DS}(\varrho)$ is a compact subset of $[0, \varrho]^{\mathrm{N}}$.

For each RAF, $\Omega$, we define

$$
\Psi_{m}(\varrho)=\inf _{\underline{r} \in D S(\varrho)} \prod_{n=0}^{\infty} \Phi_{m}(r)^{\frac{1}{2^{n+1}}}, \quad m>0 .
$$

It is proved in [22] that

$$
0<\Phi_{m}(\varrho) \leqq \Psi_{m}(\varrho)<+\infty
$$

On the other hand, since $\Phi_{m}(\varrho)$ increases as $\varrho$ decreases to 0 , the map

$$
\underline{r} \in D S(\varrho) \mapsto \prod_{n=0}^{\infty} \Phi_{m}\left(r_{n}\right)^{\frac{1}{2^{n+1}}}=\sup _{N} \prod_{n=0}^{N} \Phi_{m}\left(r_{n}\right)^{\frac{1}{2^{n+1}}}
$$

is lower semi-continuous and therefore, by the Weierstrass theorem, there exists a sequence $\tilde{r} \in D S(\varrho)$ such that

$$
\begin{gathered}
\Psi_{m}(\varrho)=\prod_{n=0}^{\infty} \Phi_{m}\left(\tilde{r}_{n}\right)^{\frac{1}{2^{n+1}}} . \\
\text { Clearly } \quad \tilde{r}_{n}>0 \text { for all } n \text { and } \sum_{n=0}^{\infty} \tilde{r}_{n}=\varrho .
\end{gathered}
$$

\subsection{Function Spaces}

In what follows we deal with functions of three parameters $(\phi, x, \mu)$, where $\phi$ runs over a domain in $\mathbb{T}+i \mathbb{R}=\mathbb{C} / \mathbb{Z}, x$ is chosen in some open neighbourhood of $\mathbb{T}$ in $\mathbb{C} / \mathbb{Z}$, and $\mu$ is a small complex parameter.

For $\phi \in \mathbb{T}+i \mathbb{R}$ we define

$$
\|\phi\|=\inf _{n \in \mathbb{Z}}|\phi+n| .
$$

For any compact subset $K$ of $\mathbb{T}+i \mathbb{R}$ and any Banach space $B$ we denote by $\mathscr{L}(K, B)$ the set of Lipschitz continuous functions from $K$ to $B$. On $\mathscr{L}(K, B)$ we define the following norm:

$$
\|F\|_{\ell}=\sup _{\phi \in K}\|F(\phi)\|+\sup _{\substack{\phi, \phi^{\prime} \in K \\ \phi \neq \phi^{\prime}}} \frac{\left\|F(\phi)-F\left(\phi^{\prime}\right)\right\|}{\left\|\phi-\phi^{\prime}\right\|} .
$$

If $B$ is a Banach algebra, $\mathscr{L}(K, B)$ turns out to be a Banach algebra under pointwise multiplication.

The following is easy to prove:

Lemma 2.1. If $B$ is a Banach algebra and $F \in \mathscr{L}(K, B)$ such that for any $\phi \in K$, $\left\|F(\phi)^{-1}\right\| \leqq k<+\infty$, then $F$ is invertible as an element of the algebra $\mathscr{L}(K, B)$ and

$$
\left\|F^{-1}\right\|_{\ell} \leqq k^{2}\|F\|_{\ell} .
$$


In what follows $B$ will be the Banach space of holomorphic bounded functions on the interior of some holomorphic manifold $\mathscr{M}$ with boundary and with values in $\mathbb{C}$ or in the two by two matrices $M_{2}(\mathbb{C})$. Here $B$ is equipped with the norm of uniform convergence. Accordingly, we shall denote $\mathscr{L}(K, B)$ by $\mathscr{L}(K, \mathscr{M}, \mathbb{C})$ or $\mathscr{L}\left(K, \mathscr{M}, M_{2}(\mathbb{C})\right)$ in these cases. More specifically $\mathscr{M}$ should be one of the following:

(i) a one point set and in which case $B=\mathbb{C}$ or $M_{2}(\mathbb{C})$,

$$
\begin{gathered}
\mathbb{T}_{r}=\mathbb{T}+i[-r, r], \quad \text { for } r>0, \\
D_{\lambda}=\{z \in \mathbb{C} ;|z| \leqq \lambda\}, \text { for } \lambda>0, \\
\mathbb{T}_{r} \times D_{\lambda} .
\end{gathered}
$$

So that there is no ambiguity, we shall drop the index $\ell$ in the norm of $\mathscr{L}(K, B)$ and denote by \|\|$,\|\|_{r},\|\|_{\lambda}$ or \|\|$_{r, \lambda}$ the norms in $\mathscr{L}\left(K, \mathscr{M}, \mathbb{C}\right.$ or $\left.M_{2}(\mathbb{C})\right)$, according to whether $\mathscr{M}$ is given by (i), (ii), (iii) or (iv).

Given an RAF $\Omega$ and an irrational $\theta$ such that

$$
\inf _{|n| \leqq m}\|n \theta\| \geqq \Omega(m),
$$

we define a domain $K_{\Omega, \theta, R}$, for $R>0$ by

$$
K_{\Omega, \theta, R}=\{\phi \in \mathbb{T}+i \mathbb{R},\|\phi+n \theta\| \geqq \Omega(|n|), n \in \mathbb{Z}\} \cap \mathbb{T}_{R} .
$$

Note that $K_{\Omega, \theta, R}$ is the complement in $\mathbb{T}_{R}$ of the union of open discs with centre $n \theta \in \mathbb{T}$ and radius $\Omega(|n|)$. If $\Omega$ decreases rapidly enough, and if $\Omega(0)$ is small enough, $K_{\Omega, \theta, R} \cap \mathbb{T}$ turns out to have non-zero Lebesgue measure.

\subsection{The Irrational Rotation}

Let $\theta \in\left[0,1\left[\right.\right.$ be an irrational number and $\left[a_{1}, a_{2}, \ldots\right]$ its continued fraction expansion. Denote, as usual,

$$
\frac{p_{n}}{q_{n}}=\left[a_{1}, a_{2}, \ldots, a_{n}\right] .
$$

Writing $\widehat{q_{n} \theta}=q_{n} \theta-p_{n}$, we see that

$$
\frac{1}{\left(a_{n+1}+2\right) q_{n}} \leqq \frac{1}{q_{n+1}+q_{n}} \leqq\left\|q_{n} \theta\right\|=\left\|\widehat{q_{n} \theta}\right\| \leqq \frac{1}{q_{n+1}} \leqq \frac{1}{a_{n+1} q_{n}} .
$$

We shall use the following results; see $[15$, p. 63] for the proofs.

Lemma 2.2. Let $q$ be such that $0<q<q_{n+1}$, then

$$
\|q \theta\| \geqq\left\|q_{n} \theta\right\| \text {. }
$$

Lemma 2.3. For each integer $j \in\left[0, q_{n+1}\left[\right.\right.$, let $\mathbf{I}_{j}^{n}$ denote the subset of $\mathbb{T}$ given by $\mathbf{I}_{j}^{n}=\left[j \theta, j \theta+\widehat{q_{n} \theta}\right](\bmod 1)$.

For any $n \geqq 1$, the $\left(q_{n}+q_{n+1}\right)$ intervals $\mathbf{I}_{j}^{n}$, for $0 \leqq j<q_{n+1}$ and $\mathbf{I}_{j}^{n+1}$ for $0 \leqq j<q_{n}$ cover $\mathbb{T}$ and have disjoint interiors. 
We say that an irrational $\theta$ is of constant type if there is a constant $c>0$ such that

$$
\left|\theta-\frac{p}{q}\right| \geqq \frac{c}{q^{2}}, \quad \forall p, q \in \mathbb{N},
$$

or, equivalently, if the continuous fraction expansion is uniformly bounded, namely $a_{n} \leqq A$, for all $n \in \mathbb{N}$. An irrational number $\theta$ is called a Liouville number if

$$
\left|\theta-\frac{p_{n}}{q_{n}}\right|<\frac{1}{\left(q_{n}\right)^{n}}
$$

The set of irrational $\theta$ for which the non-existence of localization for the Schrödinger operator related to $(1.1)$ was proved $[4,12]$ is a class of Liouville numbers, namely those for which there is a constant $c$ verifying:

$$
\left|\theta-\frac{p_{n}}{q_{n}}\right| \leqq \frac{c}{n^{q_{n}}}
$$

Note that the set of numbers of constant type as well as the set of Liouville numbers are of zero Lebesgue measure.

An irrational number is called a Roth number if, for every $\varepsilon>0$, there is a constant $c_{\varepsilon}>0$ such that

$$
\left|\theta-\frac{p}{q}\right|>\frac{c_{\varepsilon}}{q^{2+\varepsilon}}, \quad \forall p, q \in \mathbb{N} .
$$

The class of Roth numbers is of full Lebesgue measure.

\section{Statement of Results}

This section is devoted to the precise statement of the results proved in this paper. As explained before, our results agree with the André-Aubry conjecture. They will be presented in three steps. We use the notation introduced in the previous section.

\subsection{Twisted Conjugacy}

As is well known, the Schrödinger equation corresponding to (1.1) can be written as

$$
\left(\begin{array}{c}
\psi_{n+1} \\
\psi_{n}
\end{array}\right)=\left(\begin{array}{cc}
z+\mu V(x-n \theta) & -1 \\
1 & 0
\end{array}\right)\left(\begin{array}{c}
\psi_{n} \\
\psi_{n-1}
\end{array}\right) .
$$

Our first theorem asserts that, under certain circumstances, there is a twisted conjugacy of this equation to the free model. In what follows $V$ is a complexvalued continuous function on $\mathbb{T}_{r}, r>0$, holomorphic in the interior of $\mathbb{T}_{r}$ and $\|V\|_{r}$ denotes its uniform norm.

Let $\Omega$ be an RAF and $\theta$ an irrational number such that

$$
\|m \theta\| \geqq \Omega(m), \quad \forall m>0 .
$$


For a fixed $R>0$, let $K$ denote the set $K_{\Omega, \theta, R}$ defined in the previous section, see (2.21). Define a matrix-valued function by

$$
M(z, y, \mu)=\left(\begin{array}{cc}
z+\mu V(y) & -1 \\
1 & 0
\end{array}\right),
$$

where $z \in \mathbb{C}, y \in \mathbb{T}_{r}$, and $\mu \in D_{\lambda}$ for some $\lambda>0$. The corresponding free matrix is defined by

$$
M_{0}(z)=\left(\begin{array}{cc}
z & -1 \\
1 & 0
\end{array}\right)
$$

Theorem 1. There exists a constant $B>0$ such that for all $\varrho$ with $0<\varrho<r$ and all $\lambda>0$ for which

$$
\|V\|_{r} \lambda \Psi_{2}(\varrho)<B
$$

one can find a matrix-valued function $G$ in $\mathscr{L}\left(K, \mathbb{T}_{r-\varrho} \times D_{\lambda}, M_{2}(\mathbb{C})\right)$ and a complexvalued function $\alpha$ in $\mathscr{L}\left(K, D_{\lambda}, \mathbb{C}\right)$ such that

$$
M(2 \cos \pi \phi+\alpha(\phi, \mu), y, \mu)=G(\phi, y-\theta, \mu) M_{0}(2 \cos \pi \phi) \mathrm{G}(\phi, \mathrm{y}, \mu)^{-1}
$$

for all

$$
|\mu|<\lambda, \quad \phi \in K, \quad y \in \mathbb{T}_{r-\varrho} .
$$

Furthermore $G$ and $\alpha$ can be chosen in such a way that

$$
\begin{gathered}
\operatorname{det} G(\phi, y, \mu)=1, \\
\alpha(\phi, \mu=0)=0, \\
G(\phi, y, \mu=0)=\mathbb{1} .
\end{gathered}
$$

Moreover, if $V(y) \in \mathbb{R}$ for $y \in \mathbb{R}$, then for $y$ and $\mu$ real,

$$
G(\bar{\phi}, y, \mu)=\bar{G}(\phi, y, \mu) \text { and } \alpha(\bar{\phi}, \mu)=\overline{\alpha(\phi, \mu)},
$$

where $\bar{G}$ is for the complex conjugate matrix of $G$.

\subsection{Absolutely Continuous Spectrum at Small Coupling}

In addition to assumptions of (3.1), let $\Omega$ be an RAF such that

$$
\sum_{n=0}^{\infty} \Omega(n)<\frac{1}{4}
$$

and denote $K_{0}=K_{\Omega, \theta, R} \cap \mathbb{T}$.

For $x \in \mathbb{T}$, consider the self-adjoint operator on $\ell^{2}(\mathbb{Z})$, defined in (1.1):

$$
H_{x}^{(\mu)} \psi(n)=\psi(n+1)+\psi(n-1)+\mu V(x-n \theta) \psi(n) .
$$

We have the following

Theorem 2. There exists $\lambda_{1}>0$ such that for $0<\mu<\lambda_{1}$, the map

$$
E(\cdot, \mu): \phi \in K_{0} \mapsto E(\phi, \mu)=2 \cos \pi \phi+\alpha(\mu, \phi)
$$


is a Lipschitz homeomorphism which transforms $K_{0}$ into a closed subset of the spectrum of $H_{x}^{(\mu)}$ of positive Lebesgue measure; moreover, the restriction of the spectral measure of $H_{x}^{(\mu)}$ to $E\left(K_{0}, \mu\right)$ is, for any $x \in \mathbb{T}$, absolutely continuous.

\subsection{Pure Point Spectrum at High Coupling}

In addition to the assumptions of 3.1 and 3.2, we suppose now that $\theta$ is a Roth number, (see 2.3), with a continued fraction expansion

$$
\theta=\left[a_{1}, a_{2}, \ldots, a_{n}, \ldots\right]
$$

for which $\limsup _{n \rightarrow \infty} a_{n}=A(\theta) \geqq 10$. As is true for almost all irrational numbers, $A(\theta)$ may be infinite.

The following theorem insures the existence of point spectrum at high coupling for the special case of the almost Mathieu operator, namely

$$
V(x)=2 \cos 2 \pi x .
$$

Theorem 3. Under the assumptions above, there is a constant $\lambda_{2}$ such that for $\mu \geqq \lambda_{2}$ the almost Mathieu operator $H_{x}^{(\mu)}$ has, for almost all $x \in \mathbb{T}$ (with respect to Lebesgue measure), an infinite set of eigenvalues whose closure has positive Lebesgue measure. The corresponding eigenvectors have exponential decay.

More precisely, for almost all $x \in \mathbb{T}$, the set

$$
\left.K_{1}(x)=\mu E \overline{\left(K_{0} \cap\{2 x+2 n \theta, n \in \mathbb{Z}\}\right.}, \mu\right\}
$$

is in the closure of the set of eigenvalues and has positive Lebesgue measure.

\subsection{The Case where $\theta$ is of Non-Constant Type}

For a set of full Lebesgue measure, the constant

$$
A(\theta)=\limsup _{n \rightarrow \infty} a_{n}
$$

turns out to be infinite. In that case we can give an improvement on the size of the sets of the spectrum of the almost Mathieu operator described in Theorems 2 and 3. Actually in that case their Lebesgue measure is very close to that of the entire spectrum.

Theorem 4. With the same conditions as in Theorem 3, if $\theta$ is a Roth number for which $A(\theta)=\infty$, then for any $\varepsilon>0$ there is $\lambda_{0}>0$ such that, for almost all $x \in \mathbb{T}$ and $\mu \leqq \lambda_{0}$ (respectively $\mu \geqq 1 / \lambda_{0}$ ) the absolutely continuous part of the spectrum (respectively the closure of the set of eigenvalues) of the almost Mathieu operator $H_{x}^{(\mu)}$ has a Lebesgue measure greater than $4-\varepsilon$ (respectively $(4-\varepsilon) \mu$ ).

We remark that $4+4|\mu|$ is a trivial upper-bound of the Lebesgue measure of the spectrum of $H_{x}^{(\mu)}$. In [2], André and Aubry gave numerical evidence for the Lebesgue measure of the spectrum of $H_{x}^{(\mu)}$ to be $4|1-\mu|$. 


\section{The Linearized Equation}

In the proof of Theorem 1, the main point is concerned with the solution of a linearized equation and some related estimates.

Let $F$ and $G$ be given in $\mathscr{L}\left(K, \mathbb{T}_{r}, M_{2}(\mathbb{C})\right), r>0$ and let $M$ be the $2 \times 2$ matrixvalued function, defined by

$$
M(\phi)=M_{0}(2 \cos \pi \phi)=\left(\begin{array}{cc}
2 \cos \pi \phi & -1 \\
1 & 0
\end{array}\right) .
$$

As in (3.1), $\theta$ is an irrational number such that

$$
\|m \theta\| \geqq \Omega(m), \quad \forall m>0 .
$$

Associated with the equation of Theorem 1, we consider the following linearized equation:

$$
W(\phi, x-\theta) M(\phi)-M(\phi) W(\phi, x)=F(\phi, x)+G(\phi, x-\theta)^{-1} A(\phi) G(\phi, x) .
$$

The rest of the section is devoted to the proof of the existence and properties of the solutions $W$ and $A$ of this linearized equation.

Denote by $E$ the linear subspace of $M_{2}(\mathbb{C})$ of those matrices of the form

$$
\left(\begin{array}{ll}
\alpha & \beta \\
0 & 0
\end{array}\right) ; \quad \alpha, \beta \in \mathbb{C} \text {. }
$$

Proposition 5. Under the previous assumptions, there is $0<\varepsilon_{0}<1$ such that if $\|G-1\|_{r}<\varepsilon_{0}$ and $0<\varrho<r$, the linearized equation (4.3) has a solution $(W, A)$ with $W \in \mathscr{L}\left(K, \mathbb{T}_{r-\varrho}, M_{2}(\mathbb{C})\right)$ and $A \in \mathscr{L}\left(K, M_{2}(\mathbb{C})\right)$. Among all the solutions, there exists $a$ unique one such that

$$
\begin{gathered}
A(\phi) \in E, \quad \forall \phi \in K, \\
\int_{\mathbb{I}} \operatorname{tr}(W(\phi, x)) d x=0 ; \quad \int_{\mathbb{T}} \operatorname{tr}(W(\phi, x) M(\phi)) d x=0 .
\end{gathered}
$$

Furthermore, in that case, the solution satisfies

$$
\begin{gathered}
\|A\| \leqq C_{1}\|F\|_{r}, \\
\left\|F+G_{\theta}^{-1} A G\right\|_{r} \leqq C_{2}\|F\|_{r}, \\
\|W\|_{r-\varrho} \leqq C_{3} \Phi_{2}(2 \pi \varrho)\|F\|_{r},
\end{gathered}
$$

where $C_{1}, C_{2}$, and $C_{3}$ are constants depending only on $\Omega, R, \varepsilon_{0}$, and $G_{\theta}$ denotes the function

$$
G_{\theta}(\phi, x)=G(\phi, x-\theta)
$$

In order to prove this result, we first introduce for $F \in \mathscr{L}\left(K, \mathbb{T}_{r}, M_{2}(\mathbb{C})\right)$

$$
E_{n}(F)(\phi)=\int_{\mathbb{T}} d x \exp (-2 i \pi n x) F(\phi, x),
$$

and for $G \in \mathscr{L}\left(K, \mathbb{T}_{r}, M_{2}(\mathbb{C})\right), \Gamma_{G}$ denotes the operator acting on $\mathscr{L}\left(K, M_{2}(\mathbb{C})\right)$ by

$$
\Gamma_{G}(A)=E_{0}\left(G_{\theta}^{-1} A G\right) .
$$


Now the proof proceeds in three steps, the first dealing with $A$, the second with $E_{0}(W)$ and the third with $W$.

Step 1. Computation of $A$ and related estimates.

Lemma 6. Let $\eta=\|G-1\|_{r}<1$, then $\Gamma_{G}$ is bounded on $\mathscr{L}\left(K, M_{2}(\mathbb{C})\right)$ and

$$
\left\|\Gamma_{G}-\mathbb{1}\right\|_{r} \leqq 2 \eta / 1-\eta ; \quad\left\|\Gamma_{G}\right\| \leqq 1+\eta / 1-\eta .
$$

Proof. Since

$$
\left(\Gamma_{G}-\mathbb{1}\right) A=E_{0}\left(G_{\theta}^{-1} A(G-\mathbb{1})\right)+E_{0}\left(\left(G_{\theta}^{-1}-\mathbb{1}\right) A\right),
$$

and

$$
\left\|G^{-1}-\mathbb{1}\right\|_{r}=\left\|(\mathbb{1}+(G-\mathbb{1}))^{-1}-\mathbb{1}\right\|_{r} \leqq \eta / 1-\eta,
$$

we get

$$
\left\|G^{-1}\right\|_{r} \leqq 1 / 1-\eta
$$

which proves the lemma.

We now prove (a) and (b) of Proposition 5. Integrating over $x$, we see that (4.3) is equivalent to the following two equations:

$$
\operatorname{tr}\left(E_{0}(F)+\Gamma_{G}(A)\right)=0,
$$

and

$$
\operatorname{tr}\left(M E_{0}(F)+M \Gamma_{G}(A)\right)=0 .
$$

But, if $A \in E$, we get:

$$
A=-\left(\begin{array}{cc}
A_{1}(F) & A_{2}(F) \\
0 & 0
\end{array}\right)
$$

where $\left(A_{1}(F), A_{2}(F)\right)$ is the image of $\left(\operatorname{tr} E_{0}(F), \operatorname{tr} M E_{0}(F)\right)$ by the inverse $\Lambda_{G}^{-1}$ of the mapping

$$
\Lambda_{G}:(\alpha, \beta) \rightarrow\left(\operatorname{tr} \Gamma_{G}\left(\begin{array}{ll}
\alpha & \beta \\
0 & 0
\end{array}\right), \quad \operatorname{tr} M \Gamma_{G}\left(\begin{array}{ll}
\alpha & \beta \\
0 & 0
\end{array}\right)\right)
$$

Note that by Lemma $6, \Lambda_{G}^{-1}$ exists in $\mathscr{L}(K, \mathbb{C})$ provided $\|G-1\|_{r}$ is small enough. From (4.16) (a) and (b) of Proposition 5 follow immediately

Step 2. Existence and Properties of $E_{0}(W)$.

Since $E_{0}(W)$ appears only in $\left[E_{0}(W), M\right], W$ can be chosen in such a way that (ii) of Proposition 5 is verified. Then, it is a simple matter of computation that we can write:

$$
E_{0}(W)=\left(\operatorname{tr}(M)^{2}-4\right)^{-1}\left[E_{0}(F)+\Gamma_{G}(A), M\right] .
$$

Lemma 7. For $\phi \in K=K_{\Omega, \theta, R}$, we have

$$
\left\|E_{0}(W)\right\| \leqq K_{2}\|F\|_{r} .
$$

Proof. Since $4-\operatorname{tr}(M)^{2}=4 \sin ^{2} \pi \phi$ and for $|\operatorname{Re} \phi|<1 / 2$, we get $|\sin \pi \phi|>2\|\phi\|$. For $\phi \in K,\|\phi\| \geqq \Omega(0)>0$, thus $K_{2}$ exists and depends only of $\Omega(0), R,\|M\|$, and $C_{2}$. 
Step 3. Computation of $W$.

In order to compute $W$, we use a Fourier expansion and a $\phi$-dependent change of basis diagonalizing $M(\phi)$. From (4.3), we get:

$$
\exp (-2 i \pi n \theta) E_{n}(W) M(\phi)-M(\phi) E_{n}(W)=E_{n}\left(F+G_{\theta}^{-1} A G\right) .
$$

The matrix

$$
S(\phi)=\left(\begin{array}{cc}
\exp (i \pi \phi) & \exp (-i \pi \phi) \\
1 & 1
\end{array}\right)
$$

gives the desired change of basis. The following is elementary:

Lemma 8. Let $A$ be in $\mathscr{L}\left(K, M_{2}(\mathbb{C})\right)$ and let $a_{i j}$ be the matrix elements of $S(\phi)^{-1} A S(\phi)$. Then

$$
K_{3} \sup _{i, j}\left\|a_{i j}\right\| \leqq\|A\| \leqq 2 K_{3} \sup _{i, j}\left\|a_{i j}\right\|,
$$

where

$$
K_{3}=\|S(\phi)\|\left\|S(\phi)^{-1}\right\| .
$$

The rest of this section is devoted to the proof of (c) of Proposition 5. Let $W_{i j}(\phi, n)$ and $H_{i j}(\phi, n)$ be the matrix elements of $E_{n}(W)$ and of $E_{n}\left(F+G_{\theta}^{-1} A G\right)$. Writing $\varepsilon_{1}=+1$ and $\varepsilon_{2}=-1$, from (4.37), we get

$$
k=1,2, \quad n \neq 0, \quad W_{k k}(\phi, n)=\frac{H_{k h}(\phi, n) \exp \left(i \pi\left(n \theta-\varepsilon_{k} \phi\right)\right)}{2 i \sin \pi n \theta},
$$

and

$$
k \neq \ell . \quad W_{h \lambda}(\phi, n)=\frac{H_{h \lambda}(\phi, n) \exp (i \pi n())}{2 i \sin \pi\left(\varepsilon_{\ell} \phi-n \theta\right)} .
$$

Lemma 9. Let $H \in \mathscr{L}\left(K, \mathbb{T}_{r}, \mathbb{C}\right)$ and let $\|H\|_{\infty, r}$ denote the supnorm of $H$ on $K \times \mathbb{T}_{r}$. Let $\tilde{H}(\phi, n)$ be the Fourier coefficient of $H(\phi, \cdot)$ of order $n$ and let

$$
W_{\varepsilon, \alpha}(\phi, x)=\sum_{n \in \mathbb{Z}} \frac{\tilde{H}(\phi, n) \exp (2 i \pi n x)}{\sin \pi(\varepsilon \phi-n \theta)^{\alpha}},
$$

where $\varepsilon=1,0,-1$ and $\alpha \in \mathbb{N}_{*}$, then

$$
\left\|W_{\varepsilon, \alpha}\right\|_{\infty, r-\varrho} \leqq \sqrt{\pi+2} \Phi_{\alpha}(2 \pi \varrho)\|H\|_{\infty, r},
$$

for $0<\varrho<r$.

Proof. If $|\operatorname{Im} x| \leqq r-\varrho$ one gets

$$
\begin{aligned}
\left|W_{\varepsilon, \alpha}\right| & \leqq \sum_{n \in \mathbb{Z}} \frac{|\tilde{H}(\phi, n)| \exp (2 \pi|n|(r-\varrho))}{2\|\varepsilon \phi-m \phi\|^{\alpha}} \\
& \leqq \frac{1}{2} \sum_{m=0}^{\infty}\left(\sum_{p=-m}^{m} \frac{|\tilde{H}(\phi, m)| \exp (2 \pi|m| r)}{\|\varepsilon \phi-m \phi\|}\right)(\exp (-2 \pi \varrho m)-\exp (-2 \pi \varrho(m+1))) .
\end{aligned}
$$


But by Schwarz's inequality and the proposition in the appendix together with Parseval's formula

$$
\begin{aligned}
\sum_{p=-m}^{m} \frac{|\tilde{H}(\phi, m)| \exp (2 \pi|m| r)}{\|\varepsilon \phi-m \phi\|} & \leqq 2 \frac{\sqrt{\pi+2}}{\Omega(m)^{\alpha}} \int_{\mathbb{T}} d x|H(\phi, x+r)|^{2} \\
& \leqq 2 \frac{\sqrt{\pi+2}}{\Omega(m)^{\alpha}}\|H\|_{\infty, r} .
\end{aligned}
$$

Finally, since $\Omega$ is a decreasing function, we have

$$
\begin{aligned}
& \sum_{m=0}^{\infty} \frac{1}{\Omega(m)^{\alpha}}(\exp (-2 \pi \varrho m)-\exp (-2 \pi \varrho(m+1))) \\
& \quad=\sum_{m=0}^{\infty} \frac{1}{\Omega(m)^{\alpha}} \int_{2 \pi \varrho m}^{2 \pi \varrho(m+1)} e^{-s} d s \\
& \quad \leqq \int_{0}^{\infty} \frac{e^{-s}}{\Omega\left(\frac{s}{2 \pi \varrho}\right)^{\alpha}} d s=\Phi_{\alpha}(2 \pi \varrho),
\end{aligned}
$$

and (4.29) together with (4.28) proves the lemma.

Lemma 10. Under the assumptions of Lemma 9, for $0<\varrho<r$, the following inequalities hold:

For $:= \pm 1$

$$
\left\|W_{i, \alpha}\right\|_{r, \varrho} \leqq \sqrt{\pi+2}(1+\pi \alpha \operatorname{ch} \pi R) \Phi_{\alpha+1}(2 \pi \varrho)\|H\|_{r},
$$

and

$$
\left\|W_{0 . \alpha}\right\|_{r-\varrho} \leqq \sqrt{\pi+2} \omega_{\alpha}(2 \pi \varrho)\|H\|_{r} .
$$

Proof. We only need to estimate the extra term

$$
\delta W\left(\phi, \phi^{\prime}, x\right)=-\frac{W(\phi, x)-W\left(\phi^{\prime}, x\right)}{\left\|\phi-\phi^{\prime}\right\|} .
$$

But

$$
\begin{aligned}
\delta W\left(\phi, \phi^{\prime}, x\right)= & \sum_{n \in \mathbb{Z}} \exp (2 \pi n x)\left\{\frac{\delta \tilde{H}\left(\phi, \phi^{\prime}, x\right)}{\sin \pi(\varepsilon \phi-n \theta)^{\alpha}}\right. \\
& \left.-\sum_{k=1}^{\alpha} \frac{\tilde{H}\left(\phi^{\prime}, n\right) \delta\left(\sin \pi(\varepsilon-n \theta)\left(\phi, \phi^{\prime}\right)\right.}{\sin \pi(\varepsilon \phi-n \theta)^{\alpha+1-k}} \times \sin \pi(\varepsilon \phi-n \theta)^{k}\right\},
\end{aligned}
$$

where $\varepsilon= \pm 1$.

By the previous lemma and Hölder's inequality, one gets:

$$
\left|\delta W\left(\phi, \phi^{\prime}, x\right)\right| \leqq \sqrt{\pi+2}\left\{\|\delta \tilde{H}\|_{\infty, r} \Phi_{\alpha}(2 \pi \varrho)+\|H\|_{\infty, r} \pi \operatorname{ch}(\pi R \alpha) \Phi_{\alpha+1}(2 \pi \varrho)\right\},
$$

and thus

$$
\|W\|_{r-\varrho} \leqq V \overline{\pi+2}\|H\|_{r}\left\{\Phi_{\alpha}(2 \pi \varrho)+\pi(\operatorname{ch} \pi R) \alpha \Phi_{\alpha+1}(2 \pi \varrho)\right\},
$$

and since $\Omega(s)<\Omega(0) \leqq\|n \theta\| \leqq 1$ one gets $\Phi_{\alpha} \leqq \Phi_{\alpha+1}$ and (4.30) follows. 
For $\varepsilon=0$, the second part of (4.32) does not occur and so (4.30) follows directly from Lemma 9, so that Lemma 10 is proved.

We can now end up with (c) of Proposition 5. Applying Lemmas 9 and 10 to (4.23) and (4.24), we get

$$
\left\|W_{h h}\right\|_{r-\varrho} \leqq\left\|H_{h h}\right\|_{r} \exp (\pi R)\left(1+\pi \Phi_{1}(2 \pi \varrho) \sqrt{\pi+2},\right.
$$

and

$$
k \neq k^{\prime}\left\|W_{h h^{\prime}}\right\|_{r-\varrho} \leqq\left\|H_{h h^{\prime}}\right\|_{r}(1+\pi \operatorname{ch} \pi R) \Phi_{2}(2 \pi \varrho) \sqrt{\pi+2}
$$

Therefore, putting

$$
K_{4}=\operatorname{Max}(\sqrt{\pi+2}(1+\pi \operatorname{ch} \pi R), \exp (\pi R)(1+\pi) \sqrt{\pi+2}),
$$

we have

$$
\sup _{h . h^{\prime}}\left\|W_{h k^{\prime}}\right\|_{r-\varrho} \leqq K_{4} \Phi_{2}(2 \pi \varrho) \sup _{h, k^{\prime}}\left\|H_{k h^{\prime}}\right\|_{r},
$$

and finally

$$
\begin{aligned}
\left\|W-E_{0}(W)\right\|_{r-\varrho} & \leqq 2 K_{3}^{2} K_{4} \Phi_{2}(2 \pi \varrho)\|H\|_{r} \\
& \leqq 2 K_{3}^{2} K_{4} C_{2} \Phi_{2}(2 \pi \varrho)\|F\|_{r},
\end{aligned}
$$

where we use (4.8) for the last inequality.

From Lemma 9 we can write

$$
\left\|E_{0}(W)\right\| \leqq K_{2}\|F\|_{r} \leqq K_{2} \phi_{2}(2 \pi \varrho)\|F\|_{\varrho},
$$

so that, taking

$$
C_{3}=2 K_{3}^{2} K_{4}+K_{2},
$$

(4.9) follows and Proposition 5 is proved.

\section{The Recursion Process}

This section is devoted to the proof of Theorem 1 using a recursion process applied to the linearized equation. We introduce at each step matrix-valued functions $Z_{k}(\mu, \phi)$ and matrix-valued functions $G_{k}(y, \mu, \phi)$, sufficiently close to $\mathbb{1}$, so that

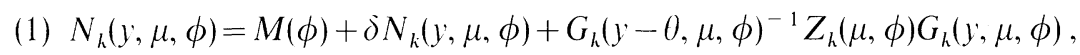

$$
\delta N_{k}(y, \mu, \theta)=O, \quad \text { as } \quad \mu \rightarrow 0,
$$

where $y \in \mathbb{T}_{r}, \mu \in D_{\lambda}$. For, we start with $Z_{0}(\mu, \phi)=0$ and $G_{0} \equiv \mathbb{1}$, so that

$$
N_{0}(y, \mu, \phi)=M(\phi)+\delta N_{0}(y, \mu, \phi),
$$

and to be explicit, we write

$$
\delta N_{0}(y, \mu, \phi)=\mu \delta N(y) .
$$


Now the results of the previous sections still hold when we replace $\mathscr{L}\left(K, \mathbb{T}_{r}, B\right)$ by $\mathscr{L}\left(K, \mathbb{T}_{r} \times D_{\lambda}, B\right), B$ being $\mathbb{C}$ or $M_{2}(\mathbb{C})$; in that case $\mu \in D_{\lambda}$ appears as an extra parameter and all the estimates are uniform in it. At each step $r$ and $\lambda$ will be made precise [see (5.18) and (5.19)].

From Proposition 5, we get $W_{k+1}(y, \mu, \phi)$ and $A_{k+1}(\mu, \phi)$ as solutions of the equation

$$
\begin{aligned}
& W_{h+1}(y-0, \mu, \phi) M(\phi)-M(\phi) W_{h+1}(y, \mu, \phi) \\
& \quad=\delta N_{h}(y, \mu, \phi)+G_{h}(y-0, \mu, \phi)^{-1} A_{h+1}(\phi, \mu) G_{h}(y, \mu, \phi) .
\end{aligned}
$$

Therefore, putting

$$
N_{h+1}(y, \mu, \phi)=\left\{1+W_{h+1}(y-0, \mu, \phi)\right\}^{-1} N_{k}(y, \mu, \phi)\left\{1+W_{k+1}(y, \mu, \phi)\right\}^{-1},
$$

it is not difficult to see that $N_{h+1}$ verifies the same relation (5.1) as $N_{h}$, with

$$
\begin{aligned}
Z_{h+1}(\mu, \phi)= & Z_{h}(\mu, \phi)-A_{h+1}(\mu, \phi), \\
G_{h+1}(1, \mu, \phi)= & G_{h}(y, \mu, \phi)\left\{1+W_{k+1}(y, \mu, \phi)\right\}, \\
\delta N_{h+1}(y, \mu, \phi)= & \left\{1+W_{h+1}(y-0, \mu, \phi)\right\}{ }^{-1}\left\{\delta N_{k}(y, \mu, \phi)\right. \\
& \left.+G_{k}(y-\theta, \mu, \phi)^{-1} A_{h+1}(\phi, \mu) G_{h}(y, \mu, \phi)\right\} W_{h+1}(y, \mu, \phi) .
\end{aligned}
$$

Following Proposition 5, we shall also get

$$
W_{k+1}(y, \mu, \phi)=O\left(\mu^{2^{k}}\right),
$$

and as $\mu \rightarrow 0$,

$$
A_{k+1}(\mu, \phi)=O\left(\mu^{2^{k}}\right),
$$

so that we can expect the recursion process to be convergent as $k \rightarrow \infty$ to some $N_{\infty}$ satisfying

$$
N_{\infty}(y, \mu, \phi)=M(\phi)+G_{\infty}(y-\theta, \mu, \phi)^{-1} Z_{\infty}(\mu, \phi) G_{\infty}(y, \mu, \phi),
$$

with

$$
G_{\infty}(y, \mu, \phi)=\prod_{k=1}^{\infty}\left(1+W_{k}(y, \mu, \phi)\right)
$$

and

$$
Z_{\infty}(\mu, \phi)=-\sum_{k=1}^{\infty} A_{k}(\mu, \phi)
$$

Now, from (5.6), we get

$$
N_{\infty}(y, \mu, \phi)=G_{\infty}(y-\theta, \mu, \phi)^{-1} N_{0}(y, \mu, \phi) G_{\infty}(y, \mu, \phi),
$$

and comparing (5.11) with (5.14), it follows that

$$
N_{0}(y, \mu, \phi)-Z_{\infty}(\mu, \phi)=G_{\infty}(y-\theta, \mu, \phi) M(\phi) G_{\infty}(y, \mu, \phi)^{-1},
$$

which will prove Theorem 1 if we are able to show that $Z_{\infty}$ is of the form

$$
\left(\begin{array}{ll}
\alpha & 0 \\
0 & 0
\end{array}\right)
$$


This is more precise than $Z_{\infty}(\mu, \phi) \in E$, which is a consequence of Proposition 5. We shall come back later to this problem.

We shall follow Rüssmann [23]. Using (2.10) and (2.11) we build a sequence $\left\{\varrho_{h}\right\}_{h \in \mathbb{N}}$ with $0<\ldots<\varrho_{k+1}<\varrho_{h}<\ldots<\varrho<r$ and $\sum_{h} \varrho_{h}=\varrho$ such that

$$
\Psi_{2}(2 \pi \varrho)=\prod_{h=0}^{3} \Phi_{2}\left(2 \pi \varrho_{h}\right)^{1 / 2^{h+1}}
$$

We define

$$
r_{k+1}=r_{k}-\varrho_{k}, \quad r_{0}=r
$$

and

$$
\lambda_{k+1}=\lambda_{k} \delta_{k}, \quad \lambda_{0}=\lambda
$$

where the $\delta_{k}, 0<\delta_{k}<1$, are chosen in the following way:

$$
\begin{aligned}
\delta_{0} & =1 \\
\left(\delta_{k}\right)^{2^{k}} & =\left(1+\varepsilon_{0}\right)^{-1} C_{2}^{-1}-\frac{\Phi\left(2 \pi \varrho_{k-1}\right)}{\Phi\left(2 \pi \varrho_{k}\right)}, \text { for } k>0,
\end{aligned}
$$

so that

$$
\prod_{k=0}^{\infty} \delta_{k}=\frac{1}{\left(1+\varepsilon_{0}\right) C_{2}} \frac{\Phi_{2}\left(2 \pi \varrho_{0}\right)}{\Psi_{2}(2 \pi \varrho)}>0
$$

From Schwarz's principle (see [23] and Proposition 5) we get the following inequalities

$$
\begin{aligned}
& 1+\left\|K_{h+1}^{-1}-\mathbb{1}\right\|_{r_{k+1} \cdot \lambda_{k+1}} \leqq\left(1+\| G_{h}^{-1}-\left.\mathbb{1}\right|_{i_{r_{k}, j_{k}}}\right)\left(1+\left\|W_{h}\right\|_{r_{k}, j_{k}}\right),
\end{aligned}
$$

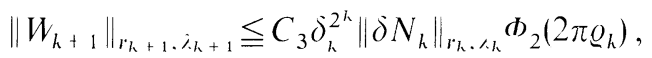

$$
\begin{aligned}
& \text { (4) } \quad\left\|\delta N_{h}\right\|_{r_{h, k}} \leqq C_{2}\left\|W_{h}\right\|_{r_{h, \lambda_{h}}}\left(1-\left\|W_{k}\right\|_{r_{k}, \lambda_{h}}\right)^{-1} \delta_{h-1}^{2 h-1}\left\|\delta N_{h-1}\right\|_{r_{h-1, l_{h-1}}} \text {. }
\end{aligned}
$$

As in [23], these recursion estimates can be solved provided

$$
\lambda_{\infty} \Psi_{2}(2 \pi \varrho)\|N\|_{r_{0}} \leqq \frac{\varepsilon_{0}}{\left(1+\varepsilon_{0}\right)^{2} C_{2} C_{3}}
$$

and $|\mu| \leqq \lambda_{\infty}=\lambda_{0} \prod_{n} \delta_{n}$. The condition on $\lambda$ in Theorem 1 follows from (5.27).

Now, in order to finish, we just need to prove that the matrix [see (5.3) and (5.13)]

$$
N_{0}(y, \mu, \phi)-Z_{\infty}(\mu, \phi)
$$

is of the form announced in Theorem 1, namely

$$
M(2 \cos \pi \phi+\alpha(\mu, \phi), y, \phi) \text {. }
$$


But, since $Z_{\infty}(\mu, \phi) \in E$, we have

$$
N_{0}(y, \mu, \phi)-Z_{\infty}(\mu, \phi)=\left(\begin{array}{cc}
2 \cos \pi \phi+\mu V(x)+\alpha(\phi, \mu) & -1+\beta(\phi, \mu) \\
1 & 0
\end{array}\right) .
$$

From (5.30) and the existence of $G_{\infty}$ we can compute

$$
\begin{aligned}
\log \operatorname{det}\left(N_{0}-Z_{\infty}\right) & =\log (1-\beta(\phi, \mu)) \\
& =\log \operatorname{det} G_{\infty}(\phi, x-\theta, \mu)-\log \operatorname{det} G_{\infty}(\phi, x, \mu) .
\end{aligned}
$$

Integrating over $x$ on $T$ one gets

$$
\log (1-\beta(\phi, \mu))=0 \quad \text { if } \quad|\mu| \leqq \lambda,
$$

or $\beta(\phi, \mu)=0$, since $\beta$ is analytical in $\mu$. Finally, it also follows that $\log \operatorname{det} G_{\infty}(\phi, x, \mu)$ is independent of $x$, and, therefore, modifying $G_{\infty}$ if necessary, we can assume

$$
\operatorname{det} G_{\infty}(\phi, x, \mu)=1 \text {, }
$$

which completes the proof of Theorem 1.

\section{Absolutely Continuous Spectrum: Proof of Theorem 2}

The matrix-valued function $G(\phi, x, \mu)$ in $\mathscr{L}\left(K, \mathbb{\pi}_{r_{\infty}} \times D_{\lambda}, M_{2}(\mathbb{C})\right)$ of determinant one, which we get by means of Theorem 1 , can be viewed as an "intertwiner" between the interacting transfer matrix $M(E(\phi, \mu), x, \mu)$ and the free transfer matrix $M_{0}(\phi)$, where

$$
E(\phi, \mu)=2 \cos \pi \phi+\alpha(\phi, \mu)
$$

provided $\phi \in K=K_{\Omega, R, \theta}$ and $|\mu|<\lambda$. From that, it is natural to define the following functions $f_{\varepsilon}$ and $g_{\varepsilon}$ in $\mathscr{L}\left(K, \mathbb{T}_{r_{\infty}} \times D_{\lambda}, \mathbb{C}\right)$, for $\varepsilon= \pm 1$, relating the solutions of the free model and such of the interacting one:

$$
\left(\begin{array}{l}
f_{\varepsilon}(\phi, x, \mu) \\
g_{\varepsilon}(\phi, x, \mu)
\end{array}\right)=G(\phi, x, \mu)^{-1}\left(\begin{array}{c}
1 \\
\exp -i \pi \varepsilon \phi
\end{array}\right) .
$$

Lemma 11. The functions $f_{\varepsilon}$ and $g_{\varepsilon}$ satisfy:

$$
g_{\varepsilon}(\phi, x, \mu)=\exp (-i \pi \varepsilon \phi) \cdot f_{\varepsilon}(\phi, x+\theta, \mu),
$$

(2) $\{E(\phi, \mu)-\mu V(x)\} \cdot f_{\varepsilon}(\phi, x, \mu)-g_{\varepsilon}(\phi, x, \mu)=\exp (i \pi \varepsilon \phi) \cdot f_{\varepsilon}(\phi, x-\theta, \mu)$.

The proof is easy.

Corollary 12. The sequences $\left\{\psi_{\varepsilon}(n)\right\}_{n \in \mathbb{Z}}, \varepsilon= \pm 1$ defined by

$$
\psi_{\varepsilon}(n)=\exp (i \pi \varepsilon n \phi) \cdot f_{\varepsilon}(\phi, x-n \theta, \mu), \quad n \in \mathbb{Z}
$$

are generalized eigenfunctions of $H_{1}^{(\mu)}$ for the eigenvalue $E(\phi, \mu)$.

Proof. Combine Lemma 11 with Theorem 1. 
Corollary 13. The Wronskian of the solutions $\psi_{+}$and $\psi_{-}$is

$$
W\left(\psi_{+}, \psi_{-}\right)=2 i \sin \pi \phi .
$$

Proof. Since the Wronskian

$$
W\left(\psi_{+}, \psi_{-}\right)=\operatorname{det}\left(\begin{array}{cc}
\psi_{+}(n) & \psi_{-}(n) \\
\psi_{+}(n-1) & \psi_{-}(n-1)
\end{array}\right)
$$

is independent of $n$, for $n=0$, we get

$$
\begin{aligned}
W\left(\psi_{+}, \psi_{-}\right) & =\operatorname{det}\left(\begin{array}{ll}
f_{+} & f_{-} \\
g_{+} & g_{-}
\end{array}\right)=\operatorname{det}\left\{G^{-1}\left(\begin{array}{cc}
1 & 1 \\
e^{-i \pi \phi} & e^{i \pi \phi}
\end{array}\right)\right\} \\
& =2 i \sin \pi \phi,
\end{aligned}
$$

where we use Lemma 11 and the fact that $\operatorname{det} G(\phi, x, \mu)=1$.

We come now to the computation of the resolvent of $H_{x}^{(\mu)}$, and first remark that

$$
\left|\psi_{i}(n)\right| \leqq \sqrt{2}\left(1+\varepsilon_{0}\right) \exp (-\varepsilon \pi n(\operatorname{Im} \phi)),
$$

since, using (6.2) and Sect. 5, we have

$$
\left\|f_{i}\right\|_{r_{\infty}, \lambda} \leqq \sqrt{2}\|G\|_{r_{\infty}, \lambda} \leqq \sqrt{2}\left(1+\varepsilon_{0}\right) .
$$

Here $\varepsilon_{0}$ is a numerical constant introduced in Proposition 5, and depending on $R$, provided they are small enough.

Lemma 14. Let $\phi \in K$ be such that $\operatorname{Im} \phi>0$, then for all $\chi \in \ell^{2}(\mathbb{Z})$, we have

$$
\begin{aligned}
\left.H_{x}^{(\mu)}-E(\phi, \mu)\right)^{-1} \chi(m)= & \frac{1}{2 i \sin \pi \phi} \\
& \cdot\left\{\psi_{+}(m) \sum_{p=-\infty}^{m} \psi_{-}(p) \chi(p)+\psi_{-}(m) \sum_{p=m+1}^{\infty} \psi_{+}(p) \chi(p)\right\} .
\end{aligned}
$$

Proof. This is a well known formula, see e.g. [27].

We now prove that if $\chi \in \ell^{1}(\mathbb{Z})$ the right hand side of $(6.10)$ remains bounded as $\operatorname{Im} \phi \rightarrow 0$.

Lemma 15. If $\chi \in \ell^{1}(\mathbb{Z}) \cap \ell^{2}(\mathbb{Z}), \phi \in K$, then

$$
\left|\left\langle\chi \mid\left(H_{\lambda}^{(\mu)}-E(\phi, \mu)\right)^{-1} \chi\right\rangle\right| \leqq\|\chi\|_{\ell}^{2} \frac{\left(1+\varepsilon_{0}\right)^{2}}{\Omega(0)} .
$$

Proof. Combining (6.9) with (6.10), one gets

$$
\begin{aligned}
\left|\psi_{+}(m) \sum_{p \leqq m} \psi_{-}(p) \chi(p)\right| & \leqq 2\left(1+\varepsilon_{0}\right) \sum_{p \leqq m} \exp (\pi \operatorname{Im} \phi(p-m))|\chi(p)| \\
& \leqq 2\left(1+\varepsilon_{0}\right)^{2}\|\chi\|_{\ell^{1}} .
\end{aligned}
$$

The remaining terms in (6.10) are bounded by the same constant and the lemma follows from the inequality:

$$
4 \Omega(0) \leqq 4\|\phi\| \leqq|2 \sin \pi \phi| .
$$


For $\phi_{0} \in K_{\Omega, \theta, R}$ and $0 \leqq \beta \leqq R$, we define two real functions $\xi^{\mu}$ and $\eta^{\mu}$ by:

$$
\xi^{\mu}\left(\phi_{0}, \beta\right)-i \eta^{\mu}\left(\phi_{0}, \beta\right)=E\left(\phi_{0}+i \beta, \mu\right)-E\left(\phi_{0}, \mu\right) .
$$

Let us remark that, by definition of $K_{\Omega, \theta, R}$ we get $\phi_{0}+i \beta \in K$.

Lemma 16. There is $a \lambda_{1}$ and $R_{0}, 0<\lambda_{1}<\lambda$ and $0<R_{0}<R$ such that, if $|\mu| \leqq \lambda_{1}$ and $0 \leqq \beta \leqq R_{0}$

$$
\begin{gathered}
\left|\xi^{\mu}\left(\phi_{0}, \beta\right)\right| \leqq 2 \pi \Omega(0) \beta, \\
\pi \beta \sin \pi \phi_{0} \leqq \eta^{\mu}\left(\phi_{0}, \beta\right) \leqq 3 \pi \beta \sin \pi \phi_{0} .
\end{gathered}
$$

Proof. Note that, by Theorem 1 , there exists $\lambda_{1}$ such that

$$
\|\alpha\|_{\lambda_{1}} \leqq \pi \Omega(0)
$$

and therefore

$$
\left|\alpha\left(\phi_{0}+i \beta, \mu\right)-\alpha\left(\phi_{0}, \mu\right)\right| \leqq \pi \Omega(0) \beta .
$$

On the other hand, by $(6.1)$ :

$$
E\left(\phi_{0}+i \beta, \mu\right)-E\left(\phi_{0}, \mu\right)=2 \cos \pi\left(\phi_{0}+i \beta\right)-2 \cos \pi \phi_{0}+\alpha\left(\phi_{0}+i \beta, \mu\right)-\alpha\left(\phi_{0}, \mu\right),
$$

from which we get:

$$
\left|\xi^{\mu}\left(\phi_{0}, \beta\right)\right| \leqq 2\left|\cos \pi \phi_{0}\right|\{\operatorname{ch} \pi \beta-1\}+\pi \Omega(0) \beta .
$$

Since we can choose $R_{0}$ so small that

$$
\operatorname{ch} \pi \beta-1 \leqq \frac{\pi}{2} \Omega(0) \beta, \quad|\beta| \leqq R_{0},
$$

(1) follows from (6.20).

In order to prove (2), we write

$$
\eta^{\mu}\left(\phi_{0}, \beta\right)=2 \sin \pi \phi_{0} \cdot \operatorname{sh} \pi \beta+\operatorname{Im}\left\{\alpha\left(\phi_{0}+i \beta, \mu\right)-\alpha\left(\phi_{0}, \mu\right)\right\} .
$$

Using $|\operatorname{sh} x-x| \leqq \frac{|x|^{3}}{3 !} \exp |x|$, we get

$$
\left|\eta^{\mu}\left(\phi_{0}, \beta\right)-2 \pi \beta \sin \pi \phi_{0}\right| \leqq \pi \Omega(0) \beta+\frac{1}{3} \pi^{3} \beta^{3} \exp (\pi \beta) \sin \pi \phi_{0} .
$$

Again by (6.13), the right hand side is dominated by $\beta \pi \sin \pi \phi_{0}$, provided $R_{0}$ is small enough to have

which proves (2).

$$
\frac{1}{3} \pi^{2} R_{0}^{2} \exp \left(\pi R_{0}\right) \leqq \frac{1}{2}
$$

Combining Lemmas 15 and 16, we easily get the following

Lemma 17. Let $\mu$ be a real number such that $0 \leqq \mu<\lambda_{1}$ and let $\sigma_{\chi}$ be the spectral measure of $H_{x}^{(\mu)}$ corresponding to the vector $\chi \in \ell^{1}(\mathbb{Z}) \cap \ell^{2}(\mathbb{Z})$. If $\phi_{0} \in K_{\Omega, \theta, R} \cap \mathbb{T}$ and $0<\beta<R_{0}$, one has

$$
\begin{aligned}
0 & \leqq \int \frac{\eta^{\mu}\left(\phi_{0}, \beta\right)}{\left\{E-E\left(\phi_{0}, \mu\right)-\xi^{\mu}\left(\Phi_{0}, \beta\right)\right\}^{2}+\eta^{\mu}\left(\phi_{0}, \beta\right)^{2}} \sigma_{\chi}(d E) \\
& \leqq\|\chi\|_{\ell^{1}}^{2} \frac{\left(1+\varepsilon_{0}\right)^{2}}{\Omega(0)} .
\end{aligned}
$$


From the previous lemma we can conclude the absolute continuity of $\sigma_{\chi}$ with essentially bounded Radon Nikodym derivative on the set $E\left(K_{\Omega, \theta, R} \cap \mathbb{T}, \mu\right)$. The proof of this property is the object of the following three lemmas.

Lemma 18. For any Borel subset $A$ of $E\left(K_{\Omega, \theta, R} \cap \mathbb{T}, \mu\right)$, we have

$$
\sigma_{\chi}(A) \leqq\|\chi\|_{\ell^{1}}^{2} 5\left(\frac{1+\varepsilon_{0}}{\Omega(0)}\right)^{2}|A| .
$$

Proof. The set $\mathscr{B}$ of measurable subsets of $\mathbb{R}$ for which (6.26) holds is a monotone family, so that we can take $A$ to be any interval centered at a point of $E\left(K_{\Omega, \theta, R} \cap \mathbb{T}, \mu\right)$, of length smaller than some fixed constant, which we take to be $4 \pi \Omega(0) R_{0}$.

But for $\phi_{0} \in K_{\Omega, \theta, R} \cap \mathbb{T}$ and $0<\beta<R_{0}$, denoting by $\mathbf{I}_{\beta}$ the interval of length $4 \pi \Omega(0) \beta$ and centered at $E\left(\phi_{n}, \mu\right)$, we can use Lemmas 16 and 17 to get

$$
\begin{aligned}
\|\chi\|_{\ell^{1}}^{2} \frac{\left(1+\varepsilon_{0}\right)^{2}}{\Omega(0)} & \geqq \int_{E \in \mathbf{I}_{\beta}} \frac{\eta^{\mu}\left(\phi_{0}, \beta\right)}{\left\{E-E\left(\phi_{0}, \mu\right)-\xi^{\mu}\left(\phi_{0}, \beta\right)\right\}^{2}+\eta^{\mu}\left(\phi_{0}, \beta\right)^{2}} \sigma_{\chi}(d E) \\
& \geqq \frac{\pi \beta \sin \left(\pi \phi_{0}\right) \sigma_{\chi}(\mathbf{I})}{16 \pi^{2} \Omega(0)^{2} \beta^{2}+9 \pi^{2} \beta^{2} \sin ^{2} \pi \phi_{0}} .
\end{aligned}
$$

From (6.27), we easily conclude:

$$
\begin{aligned}
\sigma_{\chi}(\mathbf{I}) & \leqq\|\chi\|_{\ell^{1}}^{2} \frac{\left(1+\varepsilon_{0}\right)^{2}}{\Omega(0)}|\mathbf{I}|\left(2+\frac{9}{4 \Omega(0)}\right) \\
& \leqq 5\|\chi\|_{\ell^{1}}^{2}\left(\frac{1+\varepsilon_{0}}{\Omega(0)}\right)^{2}|\mathbf{I}| .
\end{aligned}
$$

It remains to prove that $E\left(K_{\Omega, \theta, R} \cap \mathbb{T}, \mu\right)$ has positive Lebesgue measure:

Lemma 19. There is a Lipschitz homeomorphism $\hat{E}^{\mu}$ from $J=[\Omega(0), 1-\Omega(0)]$ to $\operatorname{conv} E\left(K_{\Omega, 0, R} \cap \mathbb{T}, \mu\right)$, such that

$$
\hat{E}^{\mu} \uparrow K_{\Omega, \theta, R} \cap \mathbb{T}=E(\cdot, \mu),
$$

(2) $\forall \phi \in J, \phi^{\prime} \in J$ such that $\phi \leqq \phi^{\prime}$,

$$
3 \pi \Omega(0)\left(\phi^{\prime}-\phi\right) \leqq \hat{E}^{\mu}(\phi)-\hat{E}^{\mu}\left(\phi^{\prime}\right) \leqq \pi(2+\Omega(0))\left(\phi^{\prime}-\phi\right) .
$$

Proof. If $\phi^{\prime}>\phi$ are in $J$, we have:

$$
\begin{aligned}
2 \pi\left(\phi^{\prime}-\phi\right) & \geqq 2\left(\cos \pi \phi-\cos \pi \phi^{\prime}\right) \\
& \geqq 4 \pi \Omega(0)\left(\phi^{\prime}-\phi\right) .
\end{aligned}
$$

Furthermore, if $\phi, \phi^{\prime} \in K_{\Omega, \theta, R} \cap \mathbb{T}$, we get

$$
\begin{aligned}
(2 \pi+\pi \Omega(0))\left(\phi^{\prime}-\phi\right) & \geqq\left|\alpha\left(\phi^{\prime}-\mu\right)-\alpha(\phi, \mu)\right|+2 \cos \pi \phi-2 \cos \pi \phi^{\prime} \\
& =E(\phi, \mu)-E\left(\phi^{\prime}, \mu\right) \\
& \geqq 2 \cos \pi \phi+\alpha(\phi, \mu)-2 \cos \pi \phi^{\prime}-\alpha\left(\phi^{\prime}, \mu\right) \\
& \geqq 3 \pi \Omega(0)\left(\phi^{\prime}-\phi\right),
\end{aligned}
$$

where we use (6.17). 
Now $K_{\Omega, \theta, R} \cap \mathbb{T} \subset J$ is a Cantor set and there exists a denumerable family $\left\{I_{n}\right\}_{n}$ of open disjoint intervals such that

$$
J-\left\{K_{\Omega, \theta, R} \cap \mathbb{T}\right\}=\bigcup_{n} I_{n} .
$$

If $\left.I_{n}=\right] a_{n}, b_{n}\left[\right.$ with $a_{n}, b_{n} \in K_{\Omega, \theta, R}$, we extend $E(\cdot, \mu)$ to $I_{n}$ by

$$
\hat{E}^{\mu}(\phi)=\left(\phi-a_{n}\right) E\left(a_{n}, \mu\right)-\left(b_{n}-\phi\right) E\left(b_{n}, \mu\right), \quad a_{n}<\phi<b_{n} .
$$

Since $\hat{E}^{\mu}$ again satisfies (6.32), this proves that $\hat{E}^{\mu}$ is a Lipschitz homeomorphism.

We conclude with the following:

Lemma 20. For $0 \leqq \mu \leqq \lambda_{1}$ and a subset LCT of positive Lebesgue measure, the set $E(L, \mu)$ has positive Lebesgue measure, in particular for $L=K_{\Omega, \theta, R} \cap \mathbb{T}$.

Proof. Let $\hat{F}^{\mu}$ be the inverse homeomorphism of $\hat{E}^{\mu}$. By Lemma 19(2), one has for any interval $I \subset J$ :

$$
\left|\hat{F}^{\mu}(\mathbf{I})\right| \leqq \frac{1}{3 \pi \Omega(0)}|\mathbf{I}|,
$$

and (6.35) is still true for any Borel subset of $J$; in particular

$$
|L|=\left|\hat{F}^{\mu}(E(L, \mu))\right| \leqq \frac{1}{3 \pi \Omega(0)}|E(L, \mu)|
$$

and since $\sum_{n=0}^{\infty} \Omega(n)<1 / 4$, we get

and the lemma follows.

$$
\left|K_{\Omega, \theta, R} \cap \mathbb{T}\right|>1 / 2,
$$

Now lemmas 11-20 combine to give Theorem 2.

\section{Pure Point Spectrum: Proof of Theorem 3}

We are now able to prove Theorem 3 using Aubry-André's duality [2, 6].

Proposition 21. Let $\mu$ be such that $0 \leqq \mu \leqq \lambda_{1}$ and $\phi \in \mathbb{T}$. Then for all $m \in \mathbb{Z}$ such that $\phi+2 m \theta \in K_{\Omega, 0, R}, \frac{1}{\mu} E(\phi+2 m \theta, \mu)$ is an eigenvalue of the almost Mathieu operator:

$$
\left(H_{\phi / 2}^{(1 / \mu)} \psi\right)(n)=\psi(n+1)+\psi(n-1)+\frac{2}{\mu} \cos 2 \pi\left(\frac{\phi}{2}-n \theta\right) \psi(n) .
$$

Proof. If $\phi-\phi^{\prime} \in 2 \mathbb{Z} \theta, H_{\phi / 2}^{(1 / \mu)}$ and $H_{\phi^{\prime} / 2}^{(1 / \mu)}$ are unitarily conjugate by a translation in $l^{2}(\mathbb{Z})$, therefore we only need to prove that, for $\phi \in K_{\Omega .0 . R}, \bar{\mu} E(\phi, \mu)$ is an eigenvalue of (7.1). Now by Theorem 2 and Corollary 12, the functions

$$
\left.\psi_{\varepsilon}(n)=\exp (i \varepsilon \pi n \phi) f_{\varepsilon}(\phi, x+n \theta), \mu\right), \quad \varepsilon= \pm 1
$$

are generalized eigenfunctions of $H_{x}^{(\mu)}$, provided $0 \leqq \mu \leqq \lambda_{1}$ and $\phi \in K_{\Omega, \theta, R}$ and the corresponding eigenvalue is $E(\phi, \mu)$. Therefore

$$
\left.\exp (i \pi \phi) f_{+}(x-\theta)+\exp (-i \pi \phi) f_{+}(x+\theta)+2 \mu \cos 2 \pi x-E(\phi, \mu)\right) f_{+}(x)=0 .
$$


Taking the Fourier series for $f_{+}$:

we get

$$
f_{+}(x)=\sum_{p \in \mathbb{Z}} \tilde{f}_{+}(p) \exp (2 i \pi p x)
$$

$$
E(\phi, \mu) \tilde{f}_{+}(p)=2 \cos 2 \pi\left(\frac{\phi}{2}-p \theta\right) \tilde{f}_{+}(p)+\mu\left(f_{+}(p-1)+f_{+}(p+1)\right)
$$

or

$$
H_{\phi / 2}^{(1 / \mu)} \tilde{f}_{+}=\frac{1}{\mu} E(\phi, \mu) \tilde{f}_{+}
$$

Now, we can prove Theorem 3. By Theorem 1 and Corollary 12, the functions $f_{\varepsilon}$ are analytic in $x$, in some band $|\operatorname{Im} x|<R$. Thus we get an exponential decay of the Fourier coefficients $\tilde{f}_{ \pm}(p)$ as $|p| \rightarrow \infty$.

On the other hand, Lemmas 19 and 20 tell us that $\overline{K_{0} \cap\{\phi+2 \mathbb{Z} \theta\}}$ is mapped into a set $\Xi(\phi, \mu)$ by $\phi \rightarrow \frac{1}{\mu} E(\phi, \mu)$.

The Lebesgue measure of the set $\Xi(\phi, \mu)$ is positive, provided $\overline{K_{0} \cap\{\phi+2 \mathbb{Z} \theta\}}$ is also of positive Lebesgue measure.

Under this condition, Theorem 3 is proved. Therefore the remainder of this section is devoted to the proof of the following

Proposition 22. Let $\theta=\left[a_{1}, a_{2}, \ldots\right]$ be a Roth number such that

$$
A(\theta)=\limsup _{n \geqq 1} a_{n} \geqq 10
$$

$(A(\theta)$ can be $\infty)$, and let $\Omega$ be an RAF verifying Lemma 23 with $\delta<1 / 30$.

Then, for almost all $\phi \in \mathbb{T}$ (with respect to the Lebesgue measure), the set $K_{0} \cap(\phi+2 \mathbb{Z} \theta)$ has closure with a Lebesgue measure verifying:

$$
\overline{\left|K_{0} \cap(\phi+2 \mathbb{Z} \theta)\right|} \geqq(1-6 \delta)\left(1-3 \delta-\frac{9}{A(\theta)}\right)
$$

$\left(\frac{9}{A(\theta)}\right)=0$ for $A(\theta)=\infty$.

The proof of Proposition 22 requires several steps. The first one gives a restriction on the RAF introduced previously in (2.4) and (2.5).

Lemma 23. Let $\theta$ be a Roth number - see (2.27). There exists a constant $B$ such that for any $\delta>0$, there is an $\mathrm{RAF}, \Omega$ such that

$$
\begin{gathered}
\delta \leqq B \Omega(0), \\
\Omega(0)=\Omega(1), \\
\sum_{j=1}^{\infty} \Omega(j q) \leqq \delta\|q \theta\|, \text { for all } q \in \mathbb{N} .
\end{gathered}
$$


Proof. We take $\Omega$ as in (2.4) and (2.5) for which (2) is satisfied. Now we choose $\beta$ in such a way that

$$
\Omega(s) \leqq \frac{C}{s^{\beta}}, \quad s>0
$$

By (2.27), there is a $D_{\beta}>0$ such that

$$
\|q \theta\| \geqq \frac{D_{\beta}}{q^{\beta}}
$$

so that (3) and (1) follow.

In order to estimate the Lebesgue measure of $\overline{K_{0} \cap\{\phi+2 \mathbb{Z}(0)}$ we need a good knowledge of the orbit $\phi+2 \mathbb{Z} \theta$ of $\phi$.

We define $B_{m}(m \in \mathbb{Z})$ to be the open interval of $\mathbb{T}$ centered at $m \theta(\bmod 1)$ of radius $\Omega(|m|)$, and

$$
A_{q}=\bigcup_{|m| \leqq q} B_{m}, \quad q \in \mathbb{N} .
$$

Thus, $A_{q}$ is a union of $2 q+1$ open intervals and by definition of $K_{\Omega, \theta, R} \cap \mathbb{T}=K_{0}$ we have

$$
K_{0}=\bigcap_{q \geqq 0} A_{q}^{c},
$$

where $A_{q}^{c}$ denotes the complement of $A_{q}$ in $\mathbb{T}$. Note that $\left(A_{q}^{c}\right)_{q \geqq 0}$ is a decreasing family of closed sets.

In order to localize the orbit of $\phi \in \mathbb{T}$ by the action of the irrational rotation $\theta$ we introduce the set $\mathscr{C}_{n}$ of the intervals

$$
\mathbf{I}_{j}^{n}=\left[j \theta, j \theta+\widehat{q_{n} \theta}\right] \quad(\bmod 1)
$$

such that $0 \leqq j<q_{n+1}$ and $\mathbf{I}_{j}^{n} \cap A_{q_{n}}=\emptyset$. Here $\frac{p_{n}}{q_{n}}$ is the $n$th convergent of $\theta$ and $\widehat{q_{n} \theta}=q_{n} \theta-p_{n}$.

Proposition 24. Let $n$ be positive and $\mathbf{I} \in \mathscr{C}_{n}$. Then

$$
\left|\mathbf{I} \cap K_{0}\right| \geqq(1-6 \delta)|\mathbf{I}| \text {. }
$$

In order to prove the proposition above we need the following

Lemma 25. Let $I \in \mathscr{C}_{n}$ and denote by $b_{1}$ and $b_{2}$ the balls of radius $\Omega\left(q_{n}\right)$ centered at the extremities of $\mathbf{I}$. Then, if $m \in \mathbb{Z}, m \theta \notin \mathbf{I}(\bmod 1)$, and $\mathbf{I} \cap B_{m} \neq \emptyset$, one has

$$
\mathbf{I} \cap B_{m} \subset \mathbf{I} \cap\left(b_{1} \cup b_{2}\right) .
$$

Proof. We first note that, by definition of $\mathscr{C}_{n}$, if $\mathbf{I} \cap B_{m}$ is non-empty, then $|m|>q_{n}$. Assume $\widehat{q_{n} \theta}>0$, the case $\widehat{q_{n} \theta}<0$ being treated in the same way. Then either $m \theta<j \theta(\bmod 1)$ or $m \theta>j \theta+\overline{q_{n}} \theta(\bmod 1)$. In the former case since $\Omega(|m|)<\Omega\left(q_{n}\right)$, for any $x \in \mathbf{I} \cap B_{m}$, we get

$$
x-j \theta<\Omega\left(q_{n}\right) .
$$

The latter case is treated in a similar way. 
Proof of Proposition 24. We have

$$
\mathbf{I} \cap K_{0}^{c}=\mathbf{I} \cap \bigcup_{m \in \mathbb{Z}} B_{m}=\left\{\bigcup_{m \in N_{1}}\left(\mathbf{I} \cap B_{m}\right)\right\} \cup\left\{\bigcup_{m \in N_{2}}\left(\mathbf{I} \cap B_{m}\right)\right\}
$$

where $\mathbb{Z}=N_{1} \cup N_{2}, N_{1}$ being the set of $m \in \mathbb{Z}$ for which $m \theta \notin \mathbf{I}(\bmod 1)$ and $\mathbf{I} \cap B_{m} \neq \emptyset$.

By Lemma 25 the Lebesgue measure of the first term is dominated by

$$
2 \Omega\left(q_{n}\right) \leqq 2 \delta\left\|q_{n} \theta\right\|
$$

On the other hand, using Lemma 2.2 we can write $N_{2}$ as a sequence $m_{j}, j \in Z_{*}$ in such a way that

$$
\Omega\left(\left|m_{j}\right|\right)<\Omega\left(j q_{n}\right),
$$

and by Lemma 23 the Lebesgue measure of the second term is dominated by

$$
4 \sum_{j=1}^{\infty} \Omega\left(j q_{n}\right) \leqq 4 \delta\left\|q_{n} \theta\right\|
$$

Proposition 24 follows.

Lemma 26. There exists a conull subset $N$ of $\mathbb{T}$ such that, if $\phi \in N$ and $\mathbf{I} \in \mathscr{C}_{n}(n>0)$, then

is non-empty.

$$
\{\phi+2 \mathbb{Z} \theta\} \cap \mathbf{I} \cap K_{\Omega, \theta, R}
$$

Proof. This is essentially a consequence of the individual ergodic theorem of Birkhoff applied to the map $\phi \mapsto \phi+2 \theta$ on $\mathbb{T}$.

The following is a useful division lemma for $\mathbb{T}$ :

Lemma 27. Let $A$ be the union of at most $k$ open intervals in $\mathbb{T}$ and let $\left\{\mathbf{I}_{j}\right\}_{0 \leqq j<k^{\prime}}$ be a family of closed intervals of $\mathbb{T}$ with disjoint interior and covering $\mathbb{T}$. Assume $\left|\mathbf{I}_{j}\right| \leqq \delta$, $0 \leqq j<k^{\prime}$. Then for all $k^{\prime \prime} \leqq k^{\prime}$, we have

$$
\sum_{\substack{\mathbf{I}_{j} \subset A^{c} \\ 0 \leqq j<k^{\prime \prime}}}\left|\mathbf{I}_{j}\right| \geqq\left|A^{c}\right|-\left(4 k+k^{\prime}-k^{\prime \prime}\right) \delta .
$$

Proof. Since $\left\{\mathbf{I}_{j}\right\}$ covers $\mathbb{T}$, we get

$$
1=\sum_{j=0}^{k^{\prime \prime}-1}\left|\mathbf{I}_{j}\right|+\sum_{j=k^{\prime \prime}}^{k^{\prime}-1}\left|\mathbf{I}_{j}\right|
$$

Now

$$
\sum_{j=0}^{k^{\prime \prime}-1}\left|\mathbf{I}_{j}\right|=\sum_{\substack{j \leq k^{\prime \prime}-1 \\ \overline{\mathbf{I}}_{j} \subset A}}\left|\mathbf{I}_{j}\right|+\sum_{\substack{j \leq k^{\prime \prime}-1 \\ \overline{\mathbf{I}}_{j} \subset A^{c}}}\left|\mathbf{I}_{j}\right|+\sum_{\substack{j \leq k^{\prime \prime}-1 \\ \mathbf{I}_{j} \cap \overline{\mathrm{F}}(A) \neq \emptyset}}\left|\mathbf{I}_{j}\right| .
$$

The first term of the right hand side is dominated by $|A|$. The last one is dominated by $4 k \delta$ since $\operatorname{Fr}(A)$ has at most $2 k$ points and each of these points belong at most to two of the $\mathbf{I}_{j}$. The second sum in (7.24) is dominated by $\left(k^{\prime}-k^{\prime \prime}\right) \delta$. Therefore, the lemma follows. 
We come now to the

Proof of the Proposition 22. By Lemma 26, for almost all $\phi \in \mathbb{T}$, the set Orb $\phi$ $=K_{0} \cap\{\phi+2 \mathbb{Z} \theta\}$ is non-empty. Let $U_{\varepsilon}(\phi)$ be defined by

$$
U_{\varepsilon}(\phi)=\left\{\psi \in K_{0} ; d(\psi, \operatorname{Orb} \phi) \leqq \varepsilon\right\} .
$$

Now, for $n \geqq n_{0}(\theta)$ we have $\left\|q_{n} \theta\right\| \leqq \varepsilon$ and, in that case

$$
\mathbf{I} \cap K_{\Omega, \theta, R} \subset U_{\varepsilon}(\phi)
$$

for all $\mathbf{I} \in \mathscr{C}_{n}$. Therefore, using Lemma 2.3, we get the following uniform estimate:

$$
\left|U_{\varepsilon}(\phi)\right| \geqq(1-6 \delta) \sum_{\mathbf{I} \in \mathscr{C}_{n}}|\mathbf{I}| .
$$

We use now the family of intervals $\mathbf{I}_{j}^{n}, \mathbf{I}_{j}^{n+1}$ of Lemma 2.3 in order to apply Lemma 27 to $A_{q_{n}}$ with $k^{\prime}=q_{n}+q_{n+1}$ and $k^{\prime \prime}=q_{n+1}$.

We get:

$$
\sum_{\mathbf{I} \in \mathscr{C}_{n}}|\mathbf{I}| \geqq\left|K_{0}\right|-\left(9 q_{n}+4\right)\left\|q_{n} \theta\right\| .
$$

Finally, using (2.22), we get:

$$
\limsup _{n \rightarrow \infty} \sum_{\mathbf{I} \in \mathscr{C}_{n}}|\mathbf{I}| \geqq\left|K_{0}\right|-\frac{9}{A(\theta)},
$$

and, since

$$
\left|K_{0}^{c}\right| \leqq \sum_{m \in \mathbb{Z}} 2 \Omega(|m|) \leqq 3 \delta
$$

Proposition 22 follows.

\section{The Case of Non-Constant Type: Proof of Theorem 4}

The present section is devoted to the special case of Roth numbers with $A(\theta)=\infty$, since in that case we got a better estimate of the length of the spectrum.

Let us first fix some notations. For each $\varepsilon>0$, we choose $\delta>0$ such that for a fixed $n$

$$
(1-6 \delta)(4-6 \pi \delta)>4-\frac{\varepsilon}{2}
$$

Further let $\Omega$ be an RAF as in Lemma 23 and

$$
K_{\Omega, \theta, R}, \quad K_{0}=K_{\Omega, \theta, R} \cap \mathbb{T}, \quad \text { and } \operatorname{Orb} \phi=\{\phi+2 \mathbb{Z} \theta\} \cap K_{0}
$$

as above. The main part of the proof of Theorem 4 is contained in the following:

Proposition 28. Let $\theta$ be a Roth number with $A(\theta)=\infty$ and let $E$ be the mapping

$$
E: \phi \in \mathbb{T} \rightarrow 2 \cos \pi \phi \text {. }
$$


There exists a conull set $N \subset \mathbb{T}$ such that, for $\phi \in N$, we have:

$$
\mid E \overline{(\operatorname{Orb} \phi}) \mid \geqq 4-\frac{\varepsilon}{2} .
$$

Proof. Take $N$ as in Lemma 26 and $\eta>0$. For each $\phi \in N$ we can choose $\eta_{1}>0$ such that

$$
\left|E\left(U_{\eta_{1}}\right)\right|-\eta \leqq|E(\overline{\mathrm{Orb} \phi})|,
$$

where $U_{\eta_{1}}(\phi)$ is defined in (7.26).

We now take $n$ such that (7.27) is fulfilled and also

$$
\left\|q_{n} \theta\right\|<\left\|q_{n} \theta\right\| q_{n} \leqq \eta,
$$

which is possible according to the condition on $\theta$.

Using (8.1) and (7.27), we get

$$
\eta+|E(\overline{\mathrm{Orb} \phi})| \geqq \sum_{\mathbf{I} \in \mathscr{C}_{n}} \int_{K_{0} \cap \mathbf{I}} 2 \pi \sin (\pi \phi) d \phi .
$$

For each interval $\mathbf{I}$, we can choose $\phi_{\mathbf{I}} \in \mathbf{I}$ such that

$$
\left|\sin \pi \phi-\sin \pi \phi_{\mathbf{I}}\right| \leqq \pi|\mathbf{I}|
$$

From Proposition 24 and (8.5), it follows that

$$
\begin{aligned}
\eta+|E(\overline{\mathrm{Orb} \phi})| & \geqq \sum_{\mathbf{I} \in \mathscr{C}_{n}}\left(2 \pi \sin \pi \phi_{\mathbf{I}}-2 \pi^{2}|\mathbf{I}|\right)\left(\left|\mathbf{I} \cap K_{0}\right|\right) \\
& \geqq(1-6 \delta) \sum_{\mathbf{I} \in \mathscr{C}_{n}}\left(2 \pi \sin \pi \phi_{\mathbf{I}}-2 \pi^{2}|\mathbf{I}|\right)|\mathbf{I}| .
\end{aligned}
$$

Now let $\overline{\mathscr{C}}_{n}$ be the set of intervals $\mathbf{I}_{j}^{n}, 0 \leqq j<q_{n+1}$ or $\mathbf{I}_{j}^{n+1}, 0 \leqq j<q_{n}$, for which $\mathbf{I}_{j}^{m} \cap A_{q_{n}} \neq \emptyset\left[\right.$ see (7.16)], i.e. $\mathbf{I}_{j}^{m} \notin \mathscr{C}_{n}$, then

$$
\left|4-\sum_{\mathbf{I} \in \mathscr{C}_{n}} \int_{\mathbf{I}} 2 \pi \sin (\pi \phi) d \phi\right| \leqq \sum_{\mathbf{I} \in \overline{\mathscr{C}}_{n}} \int_{\mathbf{I}} 2 \pi \sin (\pi \phi) d \phi .
$$

And, as in the proof of Lemma 27, the right hand side is divided into two parts. The first is dominated by $6 \pi \delta$ and the second by $2 \pi\left(9 q_{n}+4\right)\left\|q_{n} \theta\right\|$. It follows that, for all $\eta>0$ :

$$
\begin{aligned}
|E(\overline{\mathrm{Orb} \phi})| & \geqq(1-6 \delta)\left[4-6 \pi \delta-2 \pi\left(9 q_{n}+4\right)\right]\left\|q_{n} \theta\right\|-2 \pi^{2} q_{n+1}\left\|q_{n} \theta\right\|^{2}-\eta \\
& \geqq(1-6 \delta)\left(4-6 \pi \delta-26 \pi \eta-2 \pi^{2} \eta\right)-\eta .
\end{aligned}
$$

Proposition 28 is then a consequence of (8.1).

Proof of Theorem 4. Take $\varepsilon$ and $\delta$ as in (8.1) and choose $\lambda_{0}$ such that, for $|\mu|<\lambda_{0}$, we have - see Theorem 1 -

$$
\left|\alpha(\phi, \mu)-\alpha\left(\phi^{\prime}, \mu\right)\right| \leqq \frac{2 \pi}{B} \varepsilon \delta\left\|\phi-\phi^{\prime}\right\| ; \quad \phi, \phi^{\prime} \in K_{\Omega, \theta, R},
$$

where $B$ is the constant deduced from Lemma 23. Now

$$
2 \pi|\sin \pi \phi| \geqq 4 \pi\|\phi\| \geqq 4 \pi \frac{\delta}{B},
$$


where we use again Lemma 23 and $\|\phi\| \geqq \Omega(0)$ for $\phi \in K_{\Omega, \theta, R}$. Therefore

$$
\begin{aligned}
\left|\alpha(\phi, \mu)-\alpha\left(\phi^{\prime}, \mu\right)\right| & \leqq \frac{2 \pi}{B} \varepsilon \delta \frac{B}{4 \pi \delta}\left|E(\phi)-E\left(\phi^{\prime}\right)\right| \\
& =\frac{\varepsilon}{2}\left|E(\phi)-E\left(\phi^{\prime}\right)\right| .
\end{aligned}
$$

Take $\varepsilon \leqq \frac{1}{2}$, and therefore

$$
\frac{2 n}{B} \varepsilon \delta \leqq \pi \Omega(0)
$$

From the proof of Theorem 2, we can see that $E\left(K_{\Omega, \theta, R}, \mu\right)$ is in that case in the absolutely continuous part of the spectrum of $H_{x}^{(\mu)}$, and from the proof of Theorem 3, $1 / \mu E(\mathrm{Orb} \phi, \mu)$ in the spectrum of $H_{x}^{(1 / \mu)}$.

Now, for each Borel set $\mathbf{A}$ :

$$
E(\mathbf{A}, \mu)=E(\mathbf{A})+\alpha\left(E^{-1}(\mathbf{A}), \mu\right),
$$

and in view of (8.14), we obtain:

$$
|E(\mathbf{A}, \mu)| \geqq\left(1-\frac{\varepsilon}{2}\right)|E(\mathbf{A})| .
$$

But

$$
E(\overline{\mathrm{Orb} \phi}) \subset E\left(K_{\Omega, \theta, R}\right) .
$$

Therefore Theorem 4 follows from Proposition 28 along the same lines as in Sect. 7, replacing Proposition 22 by (8.12).

\section{Appendix}

In this appendix, we prove the following:

Proposition A.1. Let $\theta$ be an irrational number such that

$$
\sup _{0<|n| \leqq m}\|n \theta\| \geqq \Omega(m), \quad \forall m \in \mathbb{N},
$$

and let $\phi$ be a complex number such that

$$
\inf _{0 \leqq|n| \leqq m}\|n \theta+\phi\| \geqq \Omega(m), \quad \forall m \in \mathbb{N},
$$

then

$$
\sum_{n=0}^{m} \frac{1}{\|n \theta+\phi\|^{2 p}} \leqq \frac{2+\pi}{\Omega(m)^{2 p}}
$$

Here, for any complex number $z,\|z\|$ denotes the distance to the nearest integer number.

Although it is probably, well known to number theorists, we shall include a proof here for completeness since we were unable to locate a suitable reference for it. However this result is essentially an improvement of a Rüssmann estimate [24]. 
Proof. Let $p_{n} \in \mathbb{Z}$ such that

$$
\|n \theta+\phi\|=\left|n \theta+\phi-p_{n}\right| .
$$

Denote by $y_{0}<y_{1}<\ldots<y_{m}$ the $m+1$ values of $n \theta+\operatorname{Re} \phi-p_{n}, n=0,1, \ldots, m$, written in increasing order.

Since $0<\left|n_{k}-n_{\ell}\right| \leqq m$, for $k \neq \ell$, we get

$$
\left|y_{k}-y_{\ell}\right| \geqq \Omega(m) \text {. }
$$

Define $i$ by:

$$
y_{0}<\ldots<y_{i-1}<0<y_{i}<\ldots<y_{m}
$$

and $\psi=\operatorname{Im} \phi$.

Then

$$
\begin{aligned}
\sum_{n=0}^{m} & \frac{1}{\|n \theta+\phi\|^{2 p}}=\sum_{n=0}^{m} \frac{1}{\left(y_{j}^{2}+\psi^{2}\right)^{p}} \\
& =\sum_{j=0}^{i-2} \frac{1}{\left(y_{j}^{2}+\psi^{2}\right)^{p}}+\frac{1}{\left(y_{i-1}^{2}+\psi\right)^{p}}+\frac{1}{\left(y_{i}^{2}+\psi\right)^{p}}+\sum_{j=i+1}^{m} \frac{1}{\left(y_{j}^{2}+\psi^{2}\right)^{p}} .
\end{aligned}
$$

Each of the two sums of the right hand side are estimated in the same way. For instance, we have

$$
\sum_{j=i+1}^{m} \frac{1}{\left(y_{j}^{2}+\psi^{2}\right)^{p}} \leqq \frac{1}{\Omega(m)} \sum_{j=i+1}^{m} \frac{y_{j}-y_{j-1}}{\left(y_{j}^{2}+\psi^{2}\right)^{p}},
$$

and then, replacing sums by integrals, we get easily:

$$
\sum_{j=i+1}^{m} \frac{1}{\left(y_{i}^{2}+\psi^{2}\right)^{p}} \leqq \frac{\pi}{2} \frac{1}{\Omega(m)^{2 p}},
$$

from which the proposition follows.

Achnowledgements. The piesent work received, at various steps of its pieparation, the benefit of many enlightening comments of many pcople. We are happy to thank S. Albeverio, S. Aubry, G. André, J. Avron, W. Craig, G. F. Dell'Antonio, M. R. Hermann, R. Høegh-Krohn, J. Moser, E. Scoppola, B. Simon, B. Souillard, E. Trubowitz, E. Zehnder, and all our colleagues at the Centre de Physique Théorique.

Also several institutions have contributed with kind hospitality and financial support at different stages in this work. We would like to thank, in particular, the Universities of Bielefeld, Bochum, and Roma, and the California Institute of Technology.

\section{References}

1. Anderson, P.N.: Absence of diffusion in certain random lattices. Phys. Rev. 109, 1492 (1958)

2. André, G., Aubry, S.: Analyticity breaking and Anderson localization in incommensurate lattices. Ann. Israel Phys. Soc. 3, 133 (1980)

3. Arnold, V.I.: Small divisor problems in classical and celestial mechanics. Usp. Mat. Nauk. 18, $\mathrm{n}^{\circ} 6$ (114), 91-192 (1963)

4. Avron, J., Simon, B.: Singular continuous spectrum for a class of almost periodic Jacobi matrices. Bull. Am. Math. Soc. 6, 81-86 (1982)

5. Azbell, M.Ya.: Energy spectrum of a conducting electron in a magnetic field. Zh. Eksp. Teor. Fiz. 46, 929 (1964) and Sov. Phys. JETP 19, 634 (1964) 
6. Bellissard, J., Testard, D. : Almost periodic Hamiltonian : an algebraic approach. Marseille Preprint (1981)

7. Schultz, H.J., Jerome, D., Mazaud, A., Ribault, M., Bechgaard, K. : Possibility of superconducting precursor effects in quasi one-dimensional organic conductors: theory and experiments. J. Phys. (France) 42, n 7, 991-1002 (1981)

8. Bloch, A.N.: Chemical trends in organic conductors: stabilization of the nearly one-dimensional metallic state. In: Organic conductors and semi-conductors. Proceeding 1976, Pal, L., Grüner, G., Jánossy, A., Sólyon, J. (eds.). Lecture Notes in Physics, Vol. 65, p. 317. Berlin, Heidelberg, New York: Springer 1977

9. Dinaburg, E.I., Sinai, Ya.G.: The one dimensional Schrödinger equation with a quasi periodic potential. Funct. Anal. Appl. 9, 279 (1976)

10. Falicov, L.M., Hsu, W.Y.: Level quantization and broadening for band electrons in a magnetic field: magneto-optics throughout the band. Phys. Rev. B13, 1595 (1976)

11. Fröhlich, H.: On the theory of superconductivity: the one-dimensional case. Proc. R. Soc. A223, 296 (1954)

12. Gordon, A.Ya.: The point spectrum of the one-dimensional Schrödinger operator. Usp. Mat. Nauk. (Russian) 31, n 4 (190), 257 (1976)

13. Halmos, P.R.: Lectures on ergodic theory. New York: Chelsea Publ. Company 1961

14. Harper, P.G. : Single band motion of conduction electrons in a uniform magnetic field. Proc. Phys. Soc. A68, 874-892 (1955)

15. Hermann, M.R.: Sur la conjugaison différentiable des difféomorphismes du cercle à des rotations. Publ. Math. IHES n 49, 5-234 (1979)

16. Hermann, M.R.: Une méthode pour minorer les exposants de Lyapounov. Preprint (1982), Ecole Polytechnique, Palaiseau (France)

17. Hofstadter, D.R.: Energy levels and wave functions of Bloch electrons in rational and irrational magnetic fields. Phys. Rev. B14, 2239 (1976)

18. Kolmogorov, A.N.: On conservation of conditionnally periodic motion for a small change in Hamilton's function. Dokl. Akad. Nauk. SSSR (Russian) 98, 527 (1954)

Kolmogorov, A.N.: Théorie générale des systèmes dynamiques et mécanique classique. Proc. Int. Congress of Math. Amsterdam (1957)

19. Little, W.A.: Possibility of synthesizing an organic superconductor. Phys. Rev. A134, 1416 (1964)

20. Moser, J.: Convergent series expansions for quasi-periodic motions. Math. Ann. 169, 136-176 (1967)

21. Peierls, R.S.: Quantum theory of solids, Chap. VII. Oxford: Pergamon Press 1955 and Z. Phys. 80, $763(1933)$

22. Rauh, A.: Degeneracy of Landau levels in crystals. Phys. Stat. Solidi B65, K 131 (1974); B69, K 9 (1975)

23. Rüssmann, H.: On the one dimensional Schrödinger equation with a quasi-periodic potential. Ann. N. Y. Acad. Sci. 357, 90 (1980)

24. Rüssmann, H.: Note on sums containing small divisors. Commun. Pure Appl. Math. 29, 755 (1976)

25. Rüssmann, H. : On optimal estimates for the solutions of linear partial differential equations of first order with constant coefficients on the torus. In : Lecture Notes in Physics, Vol. 38, p. 598. Berlin, Heidelberg, New York: Springer 1975

26. Sarnak, P. : Spectral behaviour of quasi-periodic potential. Commun. Math. Phys. 84, 377 (1982)

27. Titchmarsh, E.C.: Eigenfunctions expansions associated with second order differential equations, Vol. 1. Oxford: Oxford University Press 1958

Communicated by B. Simon

Received May 13, 1982; in revised form June 28, 1982 